\title{
Effects of a stage-based intervention on exercise self-efficacy
}

\begin{abstract}
Studies on the changes of physical activity patterns demonstrated that physical activity rates decline sharply in adolescence and goes on well into adulthood. Psychological readiness, not physical readiness and facilities, is likely to be the main barrier to activity for most of the people. This seems to signify the importance of designing interventions to improve psychological factors related to exercise behavior willingness. This study evaluated the effect of an intervention contained strategies related to psychological factors of exercise behavior. Fifty-six sedentary adolescents completed an assessment at baseline, 2 months, and 4 months. Experimental group participants received the intervention lasting for 16 weeks and consisting of a 30-minute session every week with the aim of raising learnersôawareness about the factors affecting intention to physical activity. Control group attended physical education classes based on the guidelines of the Ministry of Education. Changes in psychological variables were compared between groups using analysis of two-way repeated measure ANOVA. The interaction between group and test was statistically significant, $\mathrm{F}(2,108)$ $=127, \mathrm{P}=.01, \mathrm{~d} 2=702)$. There was a significant difference between the scores of self-efficacy in the 3 times measurements in the experimental group. It can be concluded that the stagebased intervention had a significant positive effect on self-efficacy hence enhancing sedentary studentsôintention towards physical activity.
\end{abstract}

Keyword: Physical activity; Sedentary; Self-efficacy; Transtheoretical model 